\title{
Hartz IV aus Gender-Sicht: Einige Befunde und viele offene Fragen
}

Hat „Hartz IV“ unterschiedliche Auswirkungen auf Frauen und Männer? Bewahrheiten sich die vielfach befürchteten Benachteiligungen von Frauen durch die letzten Arbeitsmarktgesetze? Der Beitrag geht diesen Fragen anhand sekundärstatistischer Analysen nach und formuliert vorläufige Antworten im Hinblick auf Verteilungswirkungen bei Geld- und Eingliederungsleistungen. Dabei zeichnen sich tatsächlich einige geschlechtsspezifische Unterschiede ab. Offensichtlich wird jedoch auch, dass die in der amtlichen Statistik vorhandenen Datenlücken eine fundierte Analyse vor erhebliche Probleme stellen, sodass viele Fragen offen bleiben. ${ }^{1}$

\section{Einleitung}

Die Neuordnung der sozialen Sicherung gegen Arbeitslosigkeit durch das vierte „Gesetz für moderne Dienstleistungen am Arbeitsmarkt" (Hartz IV) wurde aus gleichstellungspolitischer Sicht von Beginn an überwiegend kritisch begleitet, wenn auch in ihren Wirkungen durchaus unterschiedlich bewertet. Viele Kritikerinnen bemängelten, dass besonders Frauen von der verschärften Anrechnung von Partnereinkommen durch Hartz IV betroffen seien und damit das traditionelle Ernährermodell verfestigt würde. Andere vertraten die These, die Reformen wären insgesamt geschlechterpolitisch konzeptionslos und die geschlechtsspezifischen Wirkungen blieben unklar. ${ }^{2}$ Eine weitere Einschätzung sah in den Hartz-Gesetzen eine Verallgemeinerung der prekären sozialen Bedingungen, die bisher für Frauen schon lange typisch gewesen seien. Auch der „Aktivierungsansatz" der Hartz-Gesetze ist in seinen gleichstellungspolitischen Wirkungen umstritten: Erhofft wurde, dass insbesondere benachteiligte Frauen, wie ehemalige Sozialhilfebezieherinnen, nun endlich auch von "fördernden“" Eingliederungsmaßnahmen profitieren könnten. Demgegenüber wandten Kritikerinnen ein, durch die neoliberale Ausrichtung am „männlichen“ Modell vollzeitiger Verfügbarkeit aller „erwerbsfähigen "Erwachsenen werde das feministische Ziel einer gleichberechtigten Erwerbsteilhabe nur instrumentalisiert, richte sich dabei jedoch gegen die Wünsche und Lebensmodelle vieler Frauen. Trotz der geschlechtsneutralen Formulierungen diskriminierten die Gesetze Frauen mittelbar, weil sie die unterschiedliche Lebens- und Arbeitsmarktsituation von Frauen und Männern nicht berücksichtigten. Hierzu zählen insbesondere die geschlechtshierarchische Arbeitsteilung zwischen Erwerbsarbeit und familialer Sorgearbeit, die vertikale und horizontale Segmentierung der Arbeitsmärkte, die Frauen schlechtere berufliche Positionen, niedrigere Entlohnung und eine durchschnittlich längere Arbeitslosigkeitsdauer einbringt, sowie die in die sozialen Sicherungssysteme eingelassenen Benachteiligungen typischer weiblicher Erwerbsbiografien (Bothfeld et al. 2006). Die kontroverse Bewertung der Hartz-Gesetze verweist zum einen auf ihre gleichstellungspolitische Inkonsistenz zwischen Individualisierung und Familiensubsidiarität, zum anderen auf die Notwendigkeit einer äußerst differenzierten und umfassenden empirischen Untersuchung der Wirkungen der Arbeitsmarktreformen auf unterschiedliche Gruppen von Frauen und Männern je nach individuellen Merkmalen, besonders der Haushalts- und Erwerbskonstellation und Makroindikatoren wie Region und Arbeitsmarktlage.

Eine derart differenzierte Analyse wird im Rahmen der offiziellen Gender-Evaluation zum SGB II vorgenommen, die allerdings noch am Beginn ihrer bis 2009 terminierten Arbeit steht. ${ }^{3}$ In diesem Beitrag soll dagegen nur zweieinhalb Jahre nach Inkrafttreten von Hartz IV untersucht werden, wie die wenigen aktuell verfügbaren Daten der amtlichen Statistik der Bundesagentur (BA) und des IAB unter GenderAspekten zu bewerten sind und welche weiterführenden Fragen sich daraus ergeben.

Im Hinblick auf die Gender-Bewertung dienen Kriterien als Maßstab, wie sie in der feministischen vergleichenden Wohlfahrtsstaatsforschung entwickelt wurden (Sainsbury 1999; Crompton 1998): der Grad der Unabhängigkeit von Frauen sowohl von der Versorgerehe als auch von marktvermittelter Erwerbsarbeit, der durch wohlfahrtsstaatliche Regulierungen erreicht wird. Dieser Maßstab ist im Sinne von "gender equality" dahin gehend zu erweitern, dass dieselben Kriterien auch für Männer gelten sollen. Denn angesichts allgemeiner Prekarisierungstendenzen auf Teilarbeitsmärkten ist davon auszugehen, dass diese Risiken zunehmend auch Männer betreffen und sie daher entsprechenden Sicherungsbedarf haben. Eine Gender-Bewertung von Hartz IV muss zudem den Maßstab des Gesetzes anlegen, das in $\$ 1$ Sozialgesetzbuch II (SGB II) die Gleichstellung von Frauen und Männern als „durchgängiges Prinzip “ definiert, was im Übrigen als Reaktion auf die massive Gender-Kritik im Vorfeld und auf Druck gleichstellungs-

\footnotetext{
1 Für wertvolle Hinweise bedanke ich mich bei den Teilnehmerinnen und Teilnehmern des AutorenWorkshops und den Koordinatoren dieses Heftes sowie bei Dr. Silke Bothfeld und Dr. Petra Buhr. Das Endprodukt habe ich natürlich selbst zu verantworten.

2 An dieser Stelle kann die weit verzweigte Debatte nicht nachgezeichnet werden. Einen guten systematischen Überblick bieten Rostock et al. (2007).

3 Beauftragt wurde ein Konsortium aus dem Institut für Arbeit und Qualifizierung (IAQ, Projektleitung Dr. Claudia Weinkopf), aus dem Forschungsinstitut Internationaler Arbeitsmarkt GmbH (FIA, Dr. Alexandra Wagner) und der Universität Marburg (Prof. Ingrid Kurz-Scherf, Dr. Clarissa Rudolph), www.iaq.uni-due.de.
}

Sigrid Betzelt, Dr., Diplom-Soziologin,
Zentrum für Sozialpolitik, Universität
Bremen. Arbeitsschwerpunkte: Struktur-
wandel der Erwerbsarbeit und soziale
Sicherung, wohlfahrtsstaatliche Regulierung
von Erwerbs- und Nichterwerbssphäre unter
Gender-Aspekten.
e-mail: sbetzelt@zes.uni-bremen.de


Tabelle 1: Anteil der arbeitslosen ALG-Bezieherlnnen am Bestand Arbeitsloser - in \% -

\begin{tabular}{|c|c|c|c|c|}
\hline & Westd & land & Ostc & land \\
\hline & Frauen & Männer & Frauen & Männer \\
\hline Arbeitslosengeld, JD 20041) & 40,4 & 37,3 & 25,1 & 32,2 \\
\hline Arbeitslosengeld, JD 20062) & 25,5 & 27,4 & 20,0 & 23,8 \\
\hline $\begin{array}{l}\text { Differenz JD } 04 \text { - JD } 06 \\
\text { in Prozentpunkten }\end{array}$ & $-14,9$ & $-9,9$ & $-5,1$ & $-8,4$ \\
\hline $\begin{array}{l}\text { 1) Zahlen nach Klammer } 2006, S .33 \\
\text { 2) Tabellen der BA Statistik: ALC- un } \\
\text { Hieraus wurden die relativen Anteile }\end{array}$ & herlnnen in & st, Zeitreihe & & tlicht). \\
\hline $\begin{array}{l}\text { Quelle: Berechnungen der Autorin a } \\
J D=\text { Jahresdurchschnitt. }\end{array}$ & ten in $\mathrm{Fn} 1$ & entraler Be & & ILUNGEI \\
\hline
\end{tabular}

Tabelle 2: Arbeitslosigkeit und Leistungsbezug in Deutschland, Oktober 2006

\begin{tabular}{|c|c|c|c|c|}
\hline & \multicolumn{2}{|c|}{ Frauen } & \multicolumn{2}{|c|}{ Männer } \\
\hline & absolut & in $\%$ & absolut & in \% \\
\hline Anzahl registrierte Arbeitslose & 2.051 .000 & 100 & 2.033 .000 & 100 \\
\hline Arbeitslose NichtleistungsbezieherInnen & 414.000 & 20 & 243.000 & 12 \\
\hline Arbeitslose ALG-BezieherInnen & 440.000 & 21 & 427.000 & 21 \\
\hline Arbeitslose ALG II-Bezieherlnnen & 1.250 .000 & 61 & 1.416 .000 & 70 \\
\hline Arbeitslose Leistungsbezieherlnnen insgesamt & 1.690 .000 & 82 & 1.843 .000 & 91 \\
\hline Anteil ALG an LeistungsbezieherInnen & & 26 & & 23 \\
\hline Anteil ALG II an LeistungsbezieherInnen & & 74 & & 77 \\
\hline
\end{tabular}

politischer EU-Vorgaben eingefügt wurde. Nach diesem Gleichstellungsgebot sind alle Leistungen darauf auszurichten, dass "geschlechtsspezifischen Nachteilen von erwerbsfähigen Hilfebedürftigen entgegengewirkt wird“ und „die familienspezifischen Lebensverhältnisse (...) berücksichtigt werden“" (SGB II $§ 1$ Abs. 1). Allerdings wurden, anders als im SGB III, keine diesbezüglichen Kontrollregelungen getroffen, wie das Ziel von Gender Mainstreaming konkret umgesetzt werden soll. Die im SGB III geschaffene Institution der Beauftragten für Chancengleichheit am Arbeitsmarkt ist für Arbeitslose des Rechtskreises SGB II nicht zuständig. Lediglich im Hinblick auf Eingliederungsleistungen wurden für das SGB II analog zum SGB III Frauen-Zielförderquoten festgelegt.

Zunächst wird herausgearbeitet, ob sich für bestimmte Gruppen von Frauen und Männern Verschlechterungen oder Verbesserungen durch Hartz IV hinsichtlich ihrer materiellen Lage beobachten lassen (Abschnitt 2) und wie sich die Förderung durch aktive Maßnahmen der Arbeitsmarkteingliederung nach dem Systemwechsel gestaltet (Abschnitt 3). Lässt sich daraus eine Erfüllung oder Verfehlung der gleichstellungspolitischen Ziele ableiten? Im letzten Abschnitt des Beitrags (Abschnitt 4) folgt eine abschließende Bewertung, und es werden offene Fragen sowie Datenlücken der amtlichen Statistik thematisiert. beitslosen schon vor 2004 langzeitarbeitslos und damit überwiegend im Arbeitslosenhilfebezug waren, betraf sie diese zeitliche Verkürzung seltener als westdeutsche Arbeitslose. Die überproportionale Betroffenheit westdeutscher Frauen ist zu erklären mit ihrer im Vergleich zu Männern durchschnittlich längeren Arbeitslosigkeitsdauer und ihren, verglichen mit ostdeutschen Frauen und westdeutschen Männern, geringeren Arbeitslosenhilfeansprüchen - bedingt durch das westdeutsche modernisierte Ernährermodell mit weiblichem Zuverdienst und die Anrechnung von Partnereinkommen in der Arbeitslosenhilfe. Gleichzeitig sind westdeutsche Frauen wegen ihrer häufig unterbrochenen Erwerbsbiografien stärker von dem mit Hartz III erschwerten Zugang zum ALG durch die Verkürzung der Rahmenfrist von drei auf zwei Jahre betroffen.

Längst nicht alle Arbeitslosen, die kein ALG (mehr) beziehen, haben Anspruch auf die bedarfsgeprüfte und an den Haushalt gebundene Leistung ALG II. Daten zur Verteilung Arbeitsloser auf die unterschiedlichen Leistungsarten liegen aktuell nur für Deutschland insgesamt vor, nicht aufgeschlüsselt für Ost- und Westdeutschland (Tabelle 2).

Die überwiegende Mehrheit aller registrierten Arbeitslosen bezieht ALG II, doch es zeigen sich zwei wesentliche geschlechtsspezifische Unterschiede: Frauen erhalten deutlich seltener ALG II als Männer, und fast doppelt so viele Frauen wie Männer beziehen überhaupt keine Leistungen. Der mit einem Fünftel relativ hohe Anteil an Nichtleistungsbezieherinnen ist höchstwahrscheinlich eine Folge der im Vergleich zur früheren Arbeitslosenhilfe verschärften Anrechnung von Partnereinkommen bei ALG II. Dies bestätigen auch Simulationsrechnungen von Becker/Hauser (2006). Die meisten ehemaligen Arbeitslosenhilfebezieherinnen, nämlich jene mit erwerbstätigem Partner sowie jene in Ostdeutschland, mussten überdurchschnittlich oft Einkommensverluste durch Hartz IV hinnehmen. Diese Verteilungswirkungen wurden zu Recht als mittelbare Diskriminierung gewertet, weil die Anrechnungsvorschriften in Hartz IV zwar formal für Frauen und Männer gleichermaßen gelten, indirekt aber die vorhandenen Benachteiligungen von Frauen durch Arbeitsmarktsegregation und geschlechtsspezifische Arbeitsteilung verschärfen, anstatt diese auszugleichen (Rostock et al. 2007). 
Die Analyse von Becker und Hauser identifiziert allerdings auch „GewinnerInnen“, das heißt Personengruppen, die durch den Systemwechsel potenzielle Einkommensgewinne verzeichnen konnten. Hierzu zählt insbesondere die zu $85 \%$ weibliche Gruppe Alleinerziehender, die wie in der früheren Sozialhilfe - im Sinn des SGB II überdurchschnittlich oft bedürftig ist und die besonders wegen der pauschalen Erhöhung des ALG II-Regelsatzes gegenüber der Sozialhilfe mehrheitlich höhere Leistungsansprüche verbuchen müsste. Inwieweit sich dieser Zuwachs an Leistungen allerdings tatsächlich realisiert (hat), ist ungewiss. Fraglich ist einerseits, ob die Simulationsrechnungen die reale Praxis der Leistungsgewährung widerspiegeln. Andererseits beanspruchen längst nicht alle Hilfebedürftigen tatsächlich die ihnen zustehenden Leistungen. Rund die Hälfte aller Alleinerziehenden hat ihren Hilfeanspruch nicht realisiert (Becker 2006, S. 36). Dabei handelt es sich vor allem um Erwerbstätige mit niedrigen Einkommen, die sogenannten „working poor" (Becker 2006, S. 38). Mögliche Gründe für den nicht realisierten Hilfeanspruch können mangelnde Informationen über die mit Hartz IV geschaffenen neuen Leistungsansprüche von Niedrigverdienenden sein oder auch der bewusste Verzicht auf Leistungen, um der bis in die Privatsphäre reichenden behördlichen Kontrolle zu entgehen. Diese Hintergründe wären nur durch gezielte Befragungen zu klären.

Die Studie von Becker zeigt auch, dass die Bedürftigkeitsquote von teilzeit- oder geringfügig beschäftigten Männern um zehn bis 20 Prozentpunkte über der von ebenso beschäftigten Frauen liegt (Becker 2006, S. 27). Zwar betrifft dies nur $6 \%$ der Männer gegenüber $20 \%$ der Frauen unter der ALG II-Grenze, doch die Zahlen weisen darauf hin, dass der familieninterne Lastenausgleich häufig nicht (mehr) gegeben ist und das Einverdienermodell angesichts veränderter Strukturen männlicher Erwerbstätigkeit immer weniger funktioniert.

Zusammenfassend ist festzustellen, dass

a) Frauen von dem mit Hartz IV verbundenen Systemwechsel von der lohnbezogenen Individualleistung zur bedarfsgeprüften Minimalsicherung überproportional betroffen sind, insbesondere ostdeutsche Frauen und westdeutsche ehemalige Ar- beitslosenhilfebezieherinnen mit erwerbstätigem Partner;

b) überdurchschnittlich viele Frauen vermutlich aufgrund der Anrechnung von Partnereinkommen gar keine Geldleistungen mehr erhalten;

c) im Hinblick auf potenzielle „Gewinnergruppen“ noch sehr ungewiss ist, ob z. B. Alleinerziehende von den gesetzlichen Änderungen wirklich profitieren.

Bezogen auf die angelegten Bewertungskriterien bedeuten diese vorläufigen Befunde, dass sich mit Hartz IV die eigenständige, von einem Ernährer unabhängige Existenzsicherung vieler arbeitsloser Frauen verschlechtert hat. Das gesetzliche Gleichstellungsgebot steht damit in Kontrast, besonders zu den Anrechnungsregelungen von Partnereinkommen.

\section{Verteilung von Eingliederungsleistungen}

Grundsätzlich sollte das Hauptkriterium zur Bewertung aktiver Eingliederungsleistungen die Integration in den Arbeitsmarkt sein, und zwar sinnvollerweise in existenzsichernde Erwerbsarbeit. Dies zu überprüfen, erfordert eingehende (quantitative wie qualitative) Analysen, die im Rahmen der Evaluationsforschung zu leisten sind. Hier kann nur die Verteilung von Eingliederungsleistungen auf unterschiedliche Gruppen von Arbeitslosen untersucht und dabei nach geschlechtsspezifischen Befunden sowie nach Erfüllung der gesetzlich vorgesehenen Frauen-Zielförderquoten gefragt werden. Danach sollen Frauen mindestens entsprechend ihrem Anteil an allen Arbeitslosen und ihrer relativen Betroffenheit durch Arbeitslosigkeit (Arbeitslosenquote) gefördert werden ( $\$ 16$ Abs. 1 SGB II, $\$ 8$ Abs. 2 SGB III). Die Zielförderquoten werden für arbeitslose Frauen im Rechtskreis SGB II und SGB III sowie für Ost- und Westdeutschland getrennt berechnet. Aufgrund der Datenlage kann kein Bezug zur Haushaltssituation der Arbeitslosen hergestellt werden, sodass bestimmte genderrelevante Fragen nicht zu beantworten sind (Abschnitt 4). Zur Bewertung der Art der Förderleistungen werden die Nähe der Instrumente zum ersten Arbeitsmarkt oder die dafür aufgewendeten Mittel zugrunde gelegt. Zu fragen ist
(1) inwieweit arbeitslose Frauen und Männer im Rechtskreis des SGB II in Deutschland insgesamt sowie in Ost und West durch Eingliederungsleistungen gefördert werden, und inwieweit sich dabei geschlechtsspezifische Unterschiede zeigen (Abschnit 3.1);

(2) inwieweit bestimmte Problemgruppen des Arbeitsmarktes durch Eingliederungsleistungen gefördert werden und ob es hier Gender-Disparitäten gibt (Abschnitt 3.2)

Bei der Bewertung ist außerdem der rechtliche Kontext des SGB II zu berücksichtigen. In den Blick zu nehmen sind insbesondere die fehlenden individuellen Rechtsansprüche auf Eingliederungsleistungen einerseits, andererseits die mit dem Prinzip des „Forderns“ verbundene Verpflichtung Arbeitsloser, angebotene Leistungen bei Sanktionsdrohung annehmen zu müssen.

\subsection{UNTERSCHIEDE ZWISCHEN FRAUEN UND MÄNNERN}

Arbeitslose im Rechtskreis des SGB II können sowohl mit den umfangreichen Leistungen des SGB III gefördert werden als auch mit jenen des SGB II. Betrachtet werden im Folgenden die Bestandsdaten im Jahresdurchschnitt 2005, nicht die Zu- und Abgänge, die jeweils höher liegen können, sofern es sich um kürzere Maßnahmen handelt. Aus arbeitsökonomischen und Platzgründen werden in den folgenden Tabellen nicht die Daten für Frauen und Männer ausgewiesen, sondern die absoluten und relativen Anteile geförderter Frauen im Verhältnis zu allen geförderten Arbeitslosen. ${ }^{4}$ Dies lässt ebenfalls Schlussfolgerungen auf die Gesamtförderung zu.

Tabelle 3 zeigt die absoluten und relativen Zahlen von geförderten Frauen in den sieben am häufigsten bewilligten Eingliederungsleistungen, bezogen auf alle geförderten Personen in der Bundesrepublik insgesamt sowie in West- und Ostdeutschland.

Die Gesamtbetrachtung des Niveaus der Förderung zeigt, dass nur eine kleine

\footnotetext{
4 Diese etwas ungewöhnliche Darstellungsweise ist der verfügbaren Datenstruktur der BA geschuldet, die in den Eingliederungsbilanzen Gesamtzahlen für geförderte Männer und Frauen sowie getrennte Zahlen für Frauen liefert, nicht aber auch für Männer. Um diese zu erhalten, bedarf es zusätzlicher rechnerischer Fleißarbeit.
} 


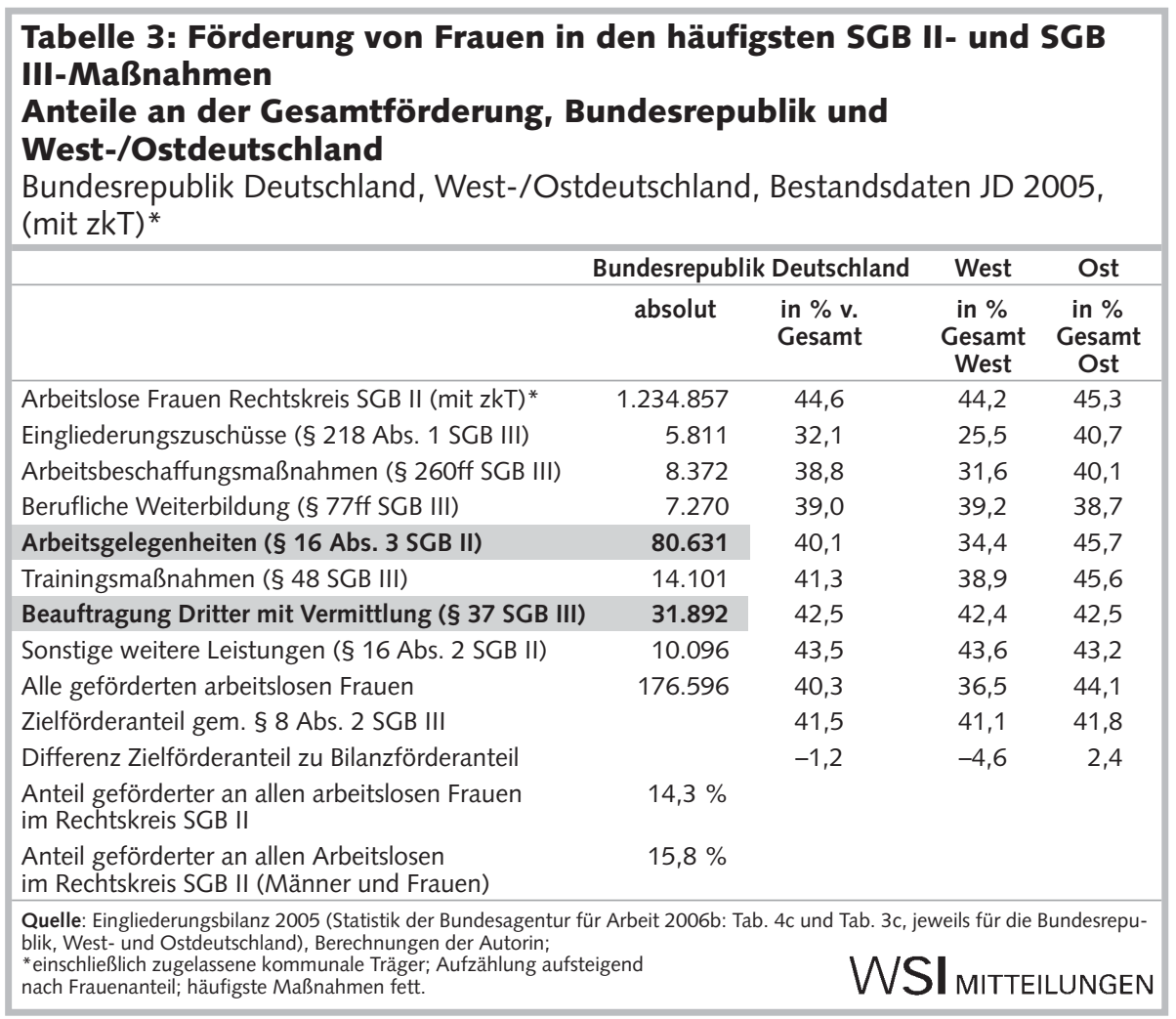

Minderheit von knapp $16 \%$ der Arbeitslosen des Rechtskreises SGB II überhaupt durch Eingliederungsmaßnahmen gefördert wird. Dieser Befund verdeutlicht das spezifisch deutsche Profil „aktivierender“ Arbeitsmarktpolitik, in dem die Komponente des „Förderns" nur schwach ausgeprägt ist gegenüber den Elementen des „Forderns“ (Dingeldey 2006).

Deutlich wird auch, dass die große Mehrheit aller Arbeitslosen, nämlich jene im Rechtskreis des SGB II, hauptsächlich mit SGB II-Maßnahmen gefördert wird, die für die BA relativ kostengünstig sind, gemessen an den durchschnittlichen ProKopf-Ausgaben pro Monat (Statistik der BA 2006b, Tab. 2). Spitzenreiter sind Arbeitsgelegenheiten („Ein-Euro-Jobs“). Seltener werden (teurere) Maßnahmen des SGB III, wie berufliche Weiterbildung oder Arbeitsbeschaffungsmaßnahmen, angewandt. Ganz besonders selten werden Leistungen gefördert, die direkt auf den ersten Arbeitsmarkt zielen, wie betriebliche Eingliederungszuschüsse.

Das Zahlenverhältnis geförderter zu arbeitslosen Frauen liegt noch unter jenem des Gesamtbestandes (14\%). Betrachtet man die Frauenanteile an den geförderten Personen insgesamt (Tabelle 3, zweite Zahlenspalte), so sind diese in den genannten vier häufigsten Maßnahmetypen am höchsten. Geschlechtsspezifische Unterschiede in

SGB III-Maßnahmen gefördert wC den als Männer, und mit einem Anteil von nur $32 \%$ besonders selten mit dem arbeitsmarktnahen Instrument betrieblicher Eingliederungszuschüsse. Im Hinblick auf die realen Eingliederungschancen ist diese geringe betriebsnahe Förderung von Frauen umso bemerkenswerter, als gerade diese zumindest für Arbeitslose im Rechtskreis SGB III - insgesamt am erfolgreichsten ist (Eingliederungsquote $70 \%$ ) und Frauen hier besonders gut abschneiden (73\%) (BA 2006b, Tab. 6a). ${ }^{5}$ Eine neue IAB-Evaluationsstudie zeigt überdies, dass der Frauenanteil an Eingliederungszuschüssen rückläufig ist, dieser lag 2001 noch bei $43 \%$ (Bernhard et al. 2007, S. 4); zugleich zeigt die Studie, dass sich die Beschäftigungschancen von Frauen durch die Förderung wesentlich verbessert haben (ebd. 2007, S. 5).

Mit 43,5 \% weisen die „Sonstigen weiteren Leistungen“ ( $\$ 16$ Abs. 2 Satz 1) den höchsten Frauenanteil auf; eine mögliche Interpretation ist, dass es sich hier vor allem um Unterstützungsleistungen bei der Kinderbetreuung handelt. Allerdings ist das absolute Niveau dieser Leistungen enorm niedrig - nur 10.000 Frauen kommen bundesweit in diesen „Genuss“. Sollte sich die BA-Unterstützung von Kinderbe- treuungsangeboten auf eine Teilmenge dieser Leistungen beschränken, dann wäre dies in der Tat ein verschwindender Beitrag, der nicht geeignet ist, den gesetzlichen Auftrag zur Verringerung geschlechtsspezifischer Nachteile und zur Berücksichtigung familienspezifischer Lebensverhältnisse zu erfüllen.

Obwohl ostdeutsche Frauen relativ häufiger als westdeutsche gefördert werden, ähnelt sich die Struktur der Unterstützung: In beiden Landesteilen werden Frauen unterproportional in arbeitsmarktnahe Maßnahmen zugewiesen, auch wenn die Relationen für ostdeutsche Frauen etwas günstiger als für westdeutsche ausfallen.

Die offizielle Zielförderquote für Frauen (SGB II) wird insgesamt mit rund einem Prozentpunkt unterschritten. ${ }^{6}$ Verfehlt wird die Quote nur in Westdeutschland, wo sie fast 5 Punkte unter dem Zielwert liegt, während sie in Ostdeutschland etwas oberhalb des Zielwerts liegt. Geschlechtsspezifische Unterschiede zeigen sich demnach weniger in der Gesamtbetrachtung für die Bundesrepublik und für Eingliederungsleistungen insgesamt, als vielmehr bei genauerer Betrachtung einzelner Maßnahmetypen und differenziert zwischen Ost und West.

\subsection{GENDER-EFFEKTE BEI „PROBLEMGRUPPEN“?}

Inwieweit werden arbeitsmarktpolitische Problemgruppen angemessen durch Eingliederungsleistungen gefördert und inwieweit zeigen sich dabei geschlechtsspezifische Unterschiede?

Ein Blick auf Tabelle 4 zeigt, dass fast alle arbeitsmarktpolitischen Problemgruppen insgesamt in der Förderung unterrepräsentiert sind. Besonders groß ist das Missverhältnis in der Gruppe der gering Qualifizierten und der Langzeitarbeitslosen, während BerufsrückkehrerInnen und Schwerbehinderte nahezu entsprechend ihres

5 Die von der BA - nur für SGB III- „Kunden“ - errechneten Eingliederungsquoten messen als Indikator für die Wirksamkeit arbeitsmarktpolitischer Maßnahmen den Anteil von Absolventen dieser Maßnahmen, die sechs Monate nach Maßnahmeende sozialversicherungspflichtig beschäftigt sind (einschließlich geförderter Beschäftigung), vgl. BA 2006, S. 125.

6 Die Zielförderquote für Frauen im Rechtskreis SGB III wird deutschlandweit mit -9 Prozentpunkten deutlicher verfehlt, wobei die Ost-West-Unterschiede geringer ausfallen (BA 2006a, S. 4) 
Anteils an Arbeitslosen gefördert werden. Die Unterrepräsentanz der Problemgruppen gilt für Frauen wie Männer. Eindeutige geschlechtsspezifische Unterschiede zeigen sich allerdings für gering qualifzierte Frauen. Sie sind in der Förderung mit knapp 12 Prozentpunkten (PP) fast doppelt so stark unterrepräsentiert wie im Gesamtbestand (knapp 7 PP).

Im Ost-West Vergleich sind zunächst Unterschiede in der Zusammensetzung der Problemgruppen festzustellen (Tabelle 5, Spalten 1 und 2, unterer Tabellenteil): Überwiegen in Westdeutschland mit rund drei Fünfteln eindeutig gering Qualifizierte, so stellen in Ostdeutschland Langzeitarbeitslose die Mehrheit. Bei der Förderung schneiden Langzeitarbeitslose im Gesamtbestand am schlechtesten ab, Ältere über 50 Jahren sind hauptsächlich in Westdeutschland unterproportional vertreten. Deutlich wird auch, dass die geschlechtsspezifische Unterrepräsentanz gering qualifizierter Frauen in der Förderung ganz überwiegend auf Westdeutschland beschränkt ist: Diese Gruppe stellt hier mit fast zwei Dritteln einen sehr hohen Anteil an arbeitslosen Frauen (Ost: ein Drittel), doch nur gut die Hälfte wird gefördert (Ost: $27,9 \%)$. Damit schneiden gering qualifizierte westdeutsche Frauen doppelt so schlecht ab wie im Gesamtbestand (Differenz arbeitslose/geförderte Frauen:-8,5 PP, Gesamt: -4 PP). Nur in den „Sonstigen weiteren Leistungen" sind sie leicht überdurchschnittlich vertreten. Festzuhalten ist damit, dass die größte und am Arbeitsmarkt besonders benachteiligte Gruppe von Frauen im alten Bundesgebiet am wenigsten gefördert wird. Die Hintergründe für diese geschlechtsspezifische Beobachtung können anhand des Datenmaterials nicht geklärt werden. Eine naheliegende Interpretation ist, dass sich in der Förderpraxis wie eventuell auch im Arbeitsmarktverhalten der Frauen traditionelle geschlechtsspezifische Muster niederschlagen. Dieser Vermutung wäre näher nachzugehen, um eventuell bestehende benachteiligende Förderstrukturen abzubauen.

Soweit zu den gesetzlich definierten arbeitsmarktpolitischen Problemgruppen. Die Gruppe Alleinerziehender zählt offiziell nicht zu diesen und wird in den Eingliederungsbilanzen nicht aufgeführt. Doch es gibt Hinweise, dass diese schon im alten Sozialhilfesystem benachteiligte Gruppe auch durch das SGB II nicht besser geför-

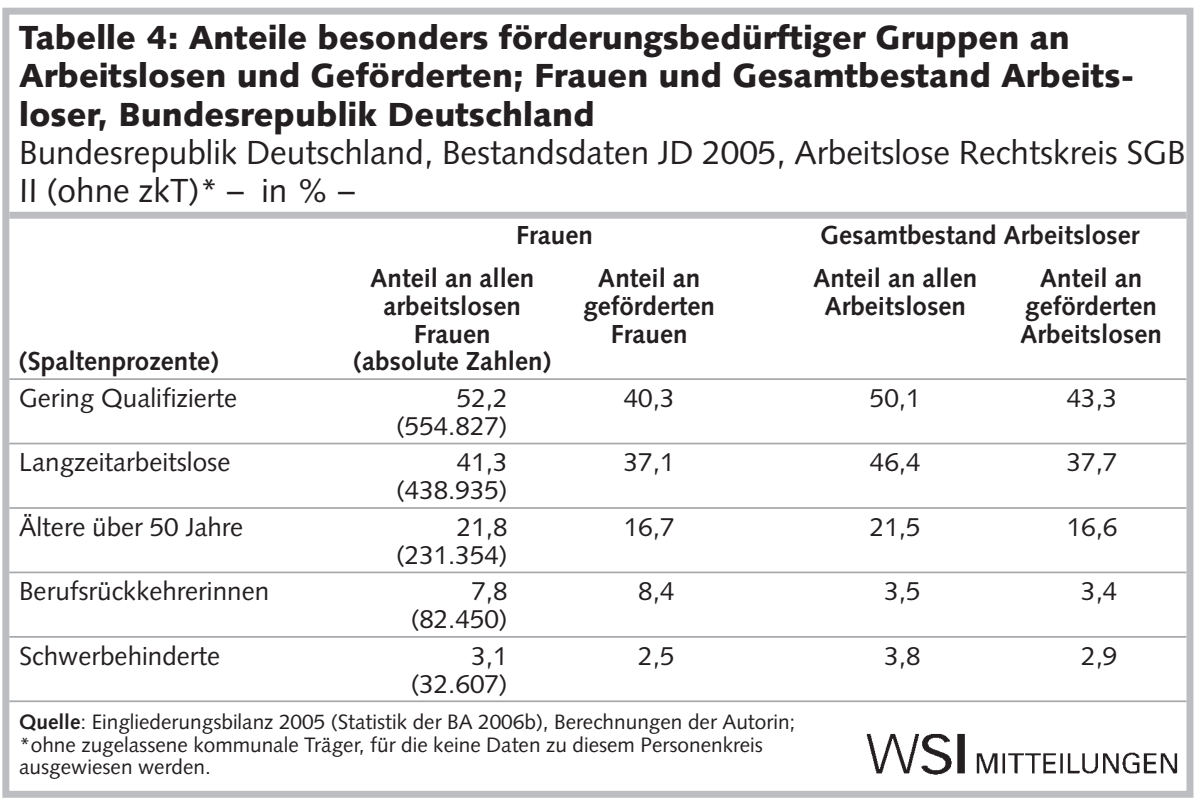

dert wird - entgegen den Erwartungen, die mit Hartz IV aus Gender-Sicht verknüpft waren.

Tabelle 6 verdeutlicht diesen Sachverhalt: Paare ohne Kinder beenden den Hilfebezug am schnellsten, Alleinerziehende sind dagegen am häufigsten dauerhaft hilfeabhängig. Damit zählen Alleinerziehende zum „niedrigdynamischen Segment" der SGB II-Population (Graf/Rudolph 2006, S. 5). Ihnen gelingt es mit dem neuen SGB II offenbar keineswegs besser als mit der alten Sozialhilfe, die Abhängigkeit vom Hilfebezug zu beenden: Der Vergleich mit der Sozialhilfe zeigt für alle Bedarfsgemeinschaften mit potenziellem SGB II-Anspruch eine Ver- bleibsquote in der Sozialhilfe von durchschnittlich $70 \%$ für 2004 (Graf/Rudolph 2006, S. 2).

Einschränkend ist anzumerken, dass sich unter den SGB II-beziehenden Alleinerziehenden nicht nur Arbeitslose befinden, sondern auch sogenannte „Aufstockerinnen", also Erwerbstätige mit niedrigen Einkommen. Leider liegen bislang keine Informationen zum Erwerbsstatus der hilfebedürftigen Alleinerziehenden vor. Letztlich liegt jedoch die soziale Problematik aller Alleinerziehenden im Zugang zu existenzsichernder Erwerbstätigkeit einerseits und der Bereitstellung adäquater Kinderbetreuung andererseits. Insofern deutet der hohe dauerhafte Verbleib im Hilfebezug

\begin{tabular}{|c|c|c|c|c|}
\hline \multicolumn{5}{|c|}{$\begin{array}{l}\text { Tabelle 5: Anteile besonders förderungsbedürftiger Gruppen an } \\
\text { Arbeitslosen und Geförderten; West-/Ostdeutschland, Frauen und } \\
\text { Gesamtbestand Arbeitsloser } \\
\text { West- /Ostdeutschland, Bestandsdaten JD 2005, Arbeitslose Rechtskreis SGB II } \\
\text { (ohne zkT)* }\end{array}$} \\
\hline & \multicolumn{2}{|c|}{ Anteil an allen arbeitslosen Frauen } & \multicolumn{2}{|c|}{ Anteil an geförderten Frauen } \\
\hline & West & Ost & West & Ost \\
\hline Gering Qualifizierte & 63,2 & 32,2 & 54,7 & 27,9 \\
\hline Langzeitarbeitslose & 34,6 & 53,5 & 27,0 & 45,7 \\
\hline Ältere über 50 Jahre & 21,5 & 22,4 & 12,9 & 19,9 \\
\hline Berufsrückkehrerinnen & 6,2 & 10,6 & 6,3 & 10,2 \\
\hline \multirow[t]{4}{*}{ Schwerbehinderte } & 3,3 & 2,6 & 2,7 & 2,4 \\
\hline & \multicolumn{4}{|c|}{ Gesamtbestand Arbeitsloser } \\
\hline & \multicolumn{2}{|c|}{ Anteil an allen Arbeitslosen } & \multicolumn{2}{|c|}{ Anteil an geförderten Arbeitslosen } \\
\hline & West & Ost & West & Ost \\
\hline Gering Qualifizierte & 59,4 & 32,6 & 55,3 & 31,0 \\
\hline Langzeitarbeitslose & 43,3 & 52,3 & 31,4 & 44,1 \\
\hline Ältere über 50 Jahre & 21,4 & 21,7 & 13,5 & 19,7 \\
\hline Berufsrückkehrerinnen & 2,8 & 4,8 & 2,3 & 4,5 \\
\hline Schwerbehinderte & 4,2 & 3,0 & 3,1 & 2,6 \\
\hline
\end{tabular}


Tabelle 6: Verbleib im SGB II-Leistungsbezug nach BG-Typ nach 12 Monaten

Verbleibsraten der Bedarfsgemeinschaften mit Zugang im Februar 2005 - in \% -

\begin{tabular}{lcc} 
& Ohne Unterbrechung & Mit Unterbrechung \\
\hline Alleinstehend & 56 & 64 \\
Alleinerziehend & 70 & 76 \\
Paar ohne Kind(er) & 48 & 60 \\
Paar mit Kind(ern) & 54 & 65 \\
Gesamt & 57 & 65 \\
\hline & & Wuelle: Graf/Rudolph 2006, S. 4, auf Basis von Daten des Administrativen Panels des IAB.
\end{tabular}

auf Defizite in der Problemlösung beider Komplexe hin. Die Problematik Alleinerziehender zeigt sich auch in ihrer überdurchschnittlichen Betroffenheit von Arbeitslosigkeit: Ihr Anteil an allen arbeitslosen Frauen (beider Rechtskreise) liegt bei 15,6 \% (Statistik der BA 2007, Tab. 3.5), gegenüber einem Anteil in der weiblichen Bevölkerung von $6 \%$ (Leiber et al. 2006, S. 47). Auf einen hohen Problemdruck verweist auch, dass die Arbeitslosigkeit in der Gruppe Alleinerziehender im Zeitverlauf von Februar 2007 zum Vorjahr nur halb so stark abnahm $(-5,6 \%)$ wie bei arbeitslosen Frauen insgesamt $(-10,6 \%)$ (Statistik der BA 2007, Tab. 3.3).

Die Vermutung liegt nahe, dass die hohe und dauerhafte Betroffenheit Alleinerziehender von Arbeitslosigkeit und SGB IIHilfebedarf auch dadurch bedingt ist, dass Erziehungspersonen von der Zumutbarkeit der Arbeitsaufnahme ausgenommen werden können ( $\$ 10$ Abs. 1 SGB II). Denn wenn die Erziehung eines Kindes nicht anders sichergestellt werden kann, wird von der Erziehungsperson die Arbeitsaufnahme nicht erwartet; generell wird dies für Kinder unter drei Jahren angenommen. Das bedeutet zugleich, dass diese Personen höchstwahrscheinlich keinerlei Angebote zur Eingliederung erhalten. Denn insgesamt entspricht die aufgezeigte Förderstruktur der Logik der Neuausrichtung der Geschäftspolitik der Bundesagentur für Arbeit (BA), nach der sich die Förderung nach betriebswirtschaftlichen Effizienzkriterien vor allem auf „starke“ Kunden konzentrieren soll - es werden vorrangig diejenigen gefördert, die die besten Vermittlungsaussichten versprechen, nicht diejenigen, bei denen der Förderbedarf am größten ist. Für besonders benachteiligte Gruppen arbeitsloser Frauen - wie z. B. gering Qualifizierte oder Alleinerziehende - erweist sich diese Ausrichtung als fatal; sie steht im Widerspruch zur SGB II-Zielsetzung, geschlechtsspezifische Benachteiligungen zu verringern.

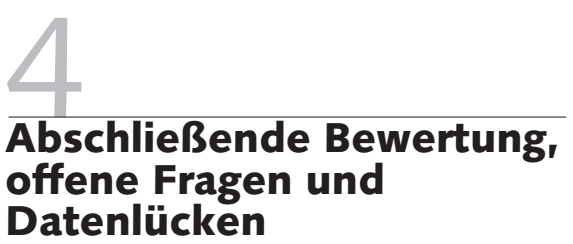

Die aus Gender-Sicht überwiegend kritisch zu bewertenden vorläufigen Befunde sind angesichts der gesetzlichen Ausgangslage von Hartz IV wenig verwunderlich. Aus einer geschlechteregalitären Perspektive enthält das SGB II zentrale "Geburtsfehler“. Dies sind vor allem die starke Verweisung auf familiale Subsidiarität durch das Konstrukt der Bedarfsgemeinschaft sowie eine ungenügende Durch- und Umsetzung von Gender Mainstreaming. Solche Konstruktionsmängel können nicht folgenlos bleiben. Die ebenfalls vom Gesetzgeber gewollte betriebswirtschaftliche Ausrichtung der neuen „aktivierenden“ Arbeitsmarktpolitik, gepaart mit fehlenden individuellen Rechtsansprüchen auf deren Leistungen, erscheint primär verantwortlich für beobachtete „creaming“-Effekte: die Konzentration der Mittel auf „starke Kunden“, die gesellschaftliche Benachteiligungen verstärkt, anstatt diese abzubauen. Beobachtet wurden dabei geschlechtsspezifische Unterschiede, denen in der weiteren Forschung nachzugehen ist, wobei besonders der Interaktionsprozess der Hilfegewährung untersucht werden muss. Wie qualitative Beobachtungen der Hartz IV-Umsetzung zeigen (Baethge-Kinsky et al. 2006; Lenhart 2006), lassen die großen Ermessensspielräume der Fachkräfte vor Ort geschlechtsrollenstereotype Annahmen wirksam werden. Die Frage nach der vollzeitigen Verfügbarkeit für den Arbeitsmarkt einerseits oder deren Einschränkung durch familiale Aufgaben andererseits kann von den Fachkräften für die einzelnen Mitglieder einer „Bedarfsgemeinschaft“ entlang traditioneller Erwartungen beantwortet werden - denn es fehlt ein Gender-Con- trolling, das dies verhindern könnte. Doch es existiert kein schlichter Automatismus: Wenn z. B. die erwerbsfähige, arbeitsmarktnah qualifizierte SGB II-„,Kundin“ eine bessere Vermittlungsfähigkeit als ihr Partner verspricht, brechen sich traditionelle Rollenerwartungen - möglicherweise des Paares selbst - am gesetzlichen Gebot eines schnellen Eingliederungserfolges. Es würde daher zu kurz greifen, Hartz IV allein eine Frauen benachteiligende Tendenz zu attestieren. Vielmehr verstärkt die prinzipielle Ausrichtung des SGB II an der Vollzeit verfügbaren Arbeitskraft ohne „care“Aufgaben (die freilich nicht konsequent durchgehalten wird, siehe die Ausnahmen von der Zumutbarkeit), neue Spaltungen innerhalb der Gruppe von Frauen und Männern - besonders entlang der beruflichen Qualifikation.

Programmiert sind derzeit jedenfalls Handlungs- und Interaktionskonflikte von Fachkräften und Adressaten der Arbeitsvermittlung, weil die Hartz IV-Umsetzung massive Eingriffe in Lebensmodelle und Vorstellungen innerfamilialer Arbeitsteilung mit sich bringt. Die Machtressourcen sind dabei sehr asymmetrisch verteilt. Anders als andere Länder des „Aktivierungsparadigmas", wie z. B. Dänemark (Dingeldey 2005), sieht das rigide deutsche Modell kaum individuelle Rechtsansprüche und wenig Handlungsautonomie für arbeitslose Hilfebeziehende vor. Aus einer geschlechteregalitären Perspektive ist aber die Stärkung der individuellen sozialen Rechte aller ErwerbsbürgerInnen unverzichtbar.

Zum Schluss muss die ausgesprochen schwierige Datenlage für eine genderspezifische Analyse thematisiert werden, ohne hier auf alle Datenlücken eingehen zu können. Das Hauptproblem besteht in der spezifischen Logik amtlicher Datenerfassung und -aufbereitung der Grundsicherungsträger (Bundesagentur für Arbeit, zugelassene kommunale Träger), die bestimmte Sachverhalte und Kategorien nicht abbildet. So werden bestimmte Personengruppen nicht gesondert erfasst bzw. in den veröffentlichten tabellarischen Übersichten zum Leistungsbezug nicht ausgewiesen. Dies gilt besonders für Arbeitslose ohne ALG II-Ansprüche und für Arbeitslose, die aufgrund des $\$ 10$ SGB II von der Zumutbarkeit ausgenommen sind (besonders Erziehungspersonen von Kindern unter drei Jahren, Alleinerziehende). Zumindest nicht bundeseinheitlich und damit nicht publi- 
kationsfähig erfasst werden auch bestimmte relevante Sachverhalte, wie die Anzahl der wegen mangelnder Bedürftigkeit abgelehnten Anträge auf ALG II. ${ }^{7}$ Gerade diese Daten wären für die Gender-Analyse höchst relevant. Wünschenswert wäre auch eine genauere Dokumentation der Ergebnisse der Vermittlungstätigkeit in Arbeitsverhältnisse auf dem „ersten“ Arbeitsmarkt im Hinblick auf Arbeitszeitumfang, Sozialversicherungspflicht oder Entlohnung der vermittelten Jobs, jeweils getrennt für Frauen und Männer.

Neben der Nichterfassung von Tatbeständen ist die Nichtverknüpfung bestimmter Kontexte ein Problem: So bestehen unterschiedliche Bezugsrahmen der Statistiken für „passive“ (Geld-) und „akti$v^{\text {" }}$ (Eingliederungs-) Leistungen. In den Statistiken zu Geldleistungen wird der Bezug zu den verschiedenen Typen von Bedarfsgemeinschaften hergestellt und damit auch die Gruppe Alleinerziehender ausge- wiesen, es fehlen aber Daten nach Einzelpersonen und Geschlecht. Dagegen beziehen sich die Eingliederungsbilanzen als Übersichten der „aktiven“ Arbeitsförderungsleistungen auf Einzelpersonen bzw. bestimmte gesetzlich definierte Problemgruppen, stellen aber keinen Bezug zur Bedarfsgemeinschaft her. Damit fehlen hier Daten zu Alleinerziehenden ebenso wie z. B. zu Personen in Paarbeziehungen oder Müttern/Vätern von Kleinkindern. Die Art und Weise der Aufbereitung der Eingliederungsbilanzen ist im Übrigen wenig benutzerfreundlich; weder werden die Zahlen für Männer und Frauen getrennt ausgewiesen, noch die relativen Anteile der besonders förderungsbedürftigen Gruppen.

Nicht zuletzt muss Gender Mainstreaming endlich so konsequent in die Statistik eingehen, dass wirklich überall, auch in Teilgruppen, wie z. B. bei MigrantInnen oder Jugendlichen, Daten für Frauen und Männer ausgewiesen werden.
Die bestehenden, teils der Gesetzeslage geschuldeten Datenlücken beschränken eine sekundärstatistische Gender-Analyse ganz erheblich; sie sind angesichts des enormen Forschungsbedarfs zum neuen Leistungssystem für Arbeitslose dauerhaft nicht hinnehmbar. Zwar ruhen große und berechtigte Hoffnungen auf der offiziellen Begleitforschung zum SGB II. Doch diese verfolgt einen dezidierten gesetzlichen Auftrag, mit dem sicher nicht alle relevanten Fragen $\mathrm{zu}$ beantworten sind. Insofern bleibt eine gendersensible Revision der amtlichen Datenerhebung und -aufbereitung ein zentrales Desiderat, das mit dem entsprechenden politischen Willen zu erfüllen wäre.

\footnotetext{
Dies ergab eine Nachfrage im Institut für Arbeitsmarkt- und Berufsforschung (IAB).
}

\section{LITERATUR}

Baethge-Kinsky, V./Bartelheimer, P./Henke, J./Land, R./Willisch, A./Wolf, A. (2006): Neue soziale Dienstleistungen nach SGB II (Konzeptstudie) - nsDL-Kon. Forschungsbericht. IAB-Projekt Nr. 823, Göttingen Becker, I. (2006): Armut in Deutschland: Bevölkerungsgruppen unterhalb der Alg II-Grenze. Arbeitspapier des Projekts "Soziale Gerechtigkeit" 3 (Oktober), Frankfurt a. M.

Becker, I./Hauser, R. (2006): Verteilungseffekte der Hartz-IV-Reform Ergebnisse von Simulationsanalysen, Berlin

Bernhard, S./Jaenichen, U./Stephan, G. (2007): Eingliederungszuschüsse: Die Geförderten profitieren, IAB Kurzbericht 9, Nürnberg Bothfeld, S./Klammer, U./Klenner, C./Leiber, S./Thiel, A./Ziegler, A. (Hrsg.) (2006): WSI FrauenDatenReport 2005 - Handbuch zur wirtschaftlichen und sozialen Situation von Frauen, Berlin

Bundesagentur für Arbeit (BA) (2006): Arbeitsmarkt 2005, in: Amtliche Nachrichten der Bundesagentur für Arbeit (ANBA) 54, Sondernummer 24.08.06

Bundesagentur für Arbeit (BA)(2006a): Statistik, Eingliederungsbilanz 2005 - Zentrale Interpretationen, Nürnberg

Bundesagentur für Arbeit (BA) (2006b): Statistik, Eingliederungsbilanz 2005 (Tabellenanhang), Nürnberg

Bundesagentur für Arbeit (BA) (2007): Statistik, Analyse des Arbeitsmarktes für Frauen und Männer, Februar 2007, Nürnberg Crompton, R. (1998): The Equality Agenda, Employment and Welfare, in: Geissler, B./Maier, F./Pfau-Effinger, B. (Hrsg.): FrauenArbeitsMarkt. Der Beitrag der Frauenforschung zur sozioökonomischen Theorieentwicklung, Berlin
Dingeldey, I. (2005): Welfare State Transformation between "Workfare“ and an "Enabling" State. A Comparative Analysis. TranState Working Papers 21, Sfb 597, "Staatlichkeit im Wandel“, Bremen

Dingeldey, I. (2006): Das Konzept des aktivierenden Staates - Paradigmenwechsel in der deutschen Arbeitsmarktpolitik. Vortrag Arbeitnehmerkammer Bremen Dezember 2006 (unveröffl. Manuskript)

Graf, T./Rudolph, H. (2006): Bedarfsgemeinschaften im SGB I| 2005 Beachtliche Dynamik bei steigenden Empfängerzahlen, IAB Kurzbericht 23, Nürnberg

Leiber, S./Thiel, A./Ziegler, A. (2006): Demografie, in: Bothfeld, S./ Klammer, U./Klenner, C./Leiber, S./Thiel, A./Ziegler, A. (Hrsg): WSI FrauenDatenReport 2005 - Handbuch zur wirtschaftlichen und sozialen Situation von Frauen, Berlin, S. 11-55

Lenhart, K. (2006): Ein "spanisches Fenster“. Erkundungen zur Frauenförderung und Hartz IV in einem großstädtischen Jobcenter, (im Erscheinen)

Rostock, P./Wersig, M./Künzel, A. (2007): Frauen diskriminierend oder geschlechterpolitisch konzeptionslos? Geschlechtsspezifische Auswirkungen von Hartz IV, in: Berghahn, S. (Hrsg.): Unterhalt und Existenzsicherung, Recht und Wirklichkeit in Deutschland, Baden-Baden, S. 305-322 Sainsbury, D. (1999) (Hrsg.): Gender and welfare state regimes, Oxford 